\title{
Approximate Chance-Constrained Unit Commitment under Wind Energy Penetration
}

\author{
Luckny Zephyr \\ Laurentian University \\ lzephyr@laurentian.ca
}

\author{
Ge Guo \\ University of Baltimore \\ cguo@ubalt.edu
}

\author{
Zongjie Wang \\ University of Connecticut \\ zongjie.wang@uconn.edu
}

\author{
José Morillo \\ Universidad del Rosario \\ jlmorill@gmail.com
}

\begin{abstract}
We study a multi-period unit commitment problem under wind energy penetration, in which the load balance is enforced with a predefined confidence level across the whole system and over the planning horizon. Since, except for special cases, chance-constrained problems are non-convex, we analyze two relaxations of the load balance based upon robust optimization ideas and estimated quantiles of the marginal distributions of the net load processes. The approximation proposals are benchmarked against the well-known scenario approximation. Under the scenario approach, we also analyze a simple decomposition strategy to find a lower bound of the approximate problem, when the latter becomes intractable due the size of the set of scenarios. The reliability of the obtained solutions as well as their runtimes are examined on three widespread test systems.
\end{abstract}

\section{Introduction}

Power systems have gone through high penetration of wind power sources thanks to their sustainability and their zero carbon emissions [1]. However, the high variability of wind speed/power may threaten the security of power system operations, and as a result, jeopardize the stability and reliability of the grid [2]. Traditional operation methods developed to address limited uncertainty in power systems, including load variations, have failed to consider the uncertainty from the unprecedented scale of wind generation outputs. As a result, advanced operation methods are required to maintain the system security and deal with wind uncertainty.

Over time, various refinements have been conducted to improve power system operation methods [3]. Under a day-ahead market framework, the system operation is considered as a two-stage problem [4], wherein in the first stage, a unit commitment (UC) problem is solved 24 hours ahead of market clearance, e.g., [5]. In a second stage, an economic dispatch problem is solved to optimize the committed controllable generation outputs toward minimizing the operating costs over a finite time frame (typically 24, 48, or 168 hours), e.g. [6].

Stochastic programming has been widely used to solve UC problems under wind uncertainty. However, a detailed analytical representation of high-dimensional random wind speed may make stochastic programs hard to solve, especially with increasing net load variability under massive integration of wind generation [7, 8]. In addition, the implicit assumption of decision makers being risk-neutral under the stochastic programming framework might fail to take extreme scenarios into account, which may jeopardize the power balancing function of the real-time dispatch, as more capacity than needed may be committed, or the opposite, over a multi-period dispatch horizon. It is therefore necessary to incorporate decision makers' risk profile in optimization models.

Robust optimization tackles this issue by seeking an optimal commitment of the traditional generators for the worst wind power scenario over a so-called uncertainty set $[8,9,10,11]$. An intermediate approach between risk-neutral stochastic programming and robust optimization, widely used in power systems, is chance-constrained programming, wherein a set of constraints is required to be met with predefined probability levels $[12,13,14,15]$. In this vein, [16] and [17] proposed chance constrained optimal power flow formulations, under renewable-based uncertainty. Multiple chance-constrained UC formulations were developed in $[13,12,18,19,20]$ to account for variable load [13], high penetration of wind [12, 18, 20, 21, 22], generator outages [19] and risk measures [18]. However, chance-constrained problems are usually non-convex [23], and as such may be challenging to solve. This has paved the way for data-driven schemes, as investigated in $[24,25,26]$.

In the same perspective, various quantile-based methods have been studied to approximate chance-constrained UC problems. In [27], a quantile-based technique is used to deal with 
the stochastic distribution of wind generation. A quantile-based iterative algorithm for chance-constrained UC problems recognizing correlated multivariate normal hourly loads was used in [13]. Another quantile approach based on the popular sample average approximation was presented in [12]. [28] used jackknife quantile estimators of the net load marginal distributions to relax the joint load balance chance-constraint.

In this paper, we study a UC problem for power systems with wind integration over a multi-period planning horizon, wherein the overall load balance is enforced with a predefined confidence level. To pass the numerical challenge induced by the non-convexity of the joint load balance chance constraint, we study two data-driven relaxation approaches based on (i) a robust-, and (ii) a quantile-based approximations of the marginal net load distributions. Our proposals are inspired by the works [29] and [13]. However, our work goes one step further, as (i) in contrast to [29], we use an iterative scheme to restore the reliability of the system as a whole, destroyed by relaxing the joint chance constraint to their individual counterparts; (ii) unlike [13], our proposal is data-driven, and as a result distribution free; and (iii) both proposals are benchmarked against the standard scenario-based approximation. In addition, as the scenario approximation approach is limited to modest size problems due to the so-called knapsack constraints, we study a simple decomposition scheme to find a lower bound of the approximate problem. Numerical case studies of three test systems with different sizes are conducted to illustrate the effectiveness and implementation of the two relaxation approaches, as well as the scenario decomposition.

In the following, a comprehensive multi-period chance-constrained UC problem with wind uncertainty is presented in Section 2. The relaxation schemes alongside the standard scenario approximation approach are discussed in Section 3. Numerical experiments and their results are provided in Section 4. Concluding remarks end the paper.

\section{Chance-Constrained Unit Commitment Problem}

Consider a power network represented as a directed graph, in which each node represents a bus $b$ indexed in the set $B$. At each node may be located a subset of conventional generators $G_{b} \subset G$, and a subset of wind farm $I_{b} \subset I$. Power outputs from the conventional generators are limited by minimum and maximum limits, denoted $\underline{P}_{g}$ and $\bar{P}_{g}$, respectively, as well as ramp-down and ramp-up capacity, denoted
$R D_{g}$ and $R U_{g}$, respectively. The UC is a multi-period problem, in which the operator of the system seeks a schedule for the conventional generators at minimum cost, - including production, start-up, shut-down, and reserve cost of each generator $g$ in each period $t \in$ $T$, denoted $c_{g}^{t}, c s_{g}^{t}, c d_{g}^{t}$, and $c r_{g}^{t}$, respectively. This schedule is sought to satisfy the load across the system, under operating constraints.

More specifically, in each period $t$, the UC problem seeks the on/off, start-up, and shut-down status of the conventional generators, denoted $u_{g}^{t}, \nu_{g}^{t}$, and $\mu_{g}^{t}$, respectively, their production level $p_{g}^{t}$, their contribution, $r_{g}^{t}$, to the required spinning reserves, $\alpha^{t}$, taken as a fraction of the total load. In addition, in each period $t$, the power flowing between end buses $i$, and $j, f_{i, j}^{t}$, through the transmission line $l_{i, j}:=(i, j) \in L$, is proportional to the susceptance of the line, $B_{i, j}$, and the difference between the voltage angles at the two buses, $\theta_{i}^{t}-\theta_{j}^{t}$, and is bounded by the line capacity, $\bar{F}_{i, j}$.

Lastly, we assume that in each period $t$, the load at bus $b$ is forecasted at $L_{b}^{t}$, and the wind power at $\sum_{i \in I_{b}} W_{i}^{t}$. However, since wind speed/power is highly unpredictable, we shall enforce that the load be met with probability no less than a chosen confidence level, $\rho$, over the planing horizon and across the network. Thus, our UC problem may be formulated as the following chance-constrained optimization program:

$$
\min \sum_{g \in G} \sum_{t \in T}\left(c_{g}^{t}+c s_{g}^{t}+c d_{g}^{t}+c r_{g}^{t}\right)
$$

subject to: $c_{g}^{t} \geq C U_{g} u_{g}^{t}+C P_{g} p_{g}^{t}, g \in G, t \in T$,

$c s_{g}^{t} \geq C S_{g} v_{g}^{t}, g \in G, t \in T$,

$c d_{g}^{t} \geq C D_{g} \mu_{g}^{t}, g \in G, t \in T$,

$c r_{g}^{t} \geq C R_{g} r_{g}^{t}, g \in G, t \in T$,

$\mathbb{P}\left(\sum_{g \in G_{b}}\left(p_{g}^{t}+r_{g}^{t}\right)+\sum_{l_{i, b} \in L} f_{i, b}^{t}-\right.$

$$
\begin{aligned}
& \sum_{l_{b, j} \in L} f_{b, j}^{t} \geq L_{b}^{t}-\sum_{i \in I_{b}} W_{i}^{t}, \\
&b \in B, t \in T) \geq \rho,
\end{aligned}
$$

$u_{g}^{t} \underline{P}_{g} \leq p_{g}^{t}+r_{g}^{t} \leq u_{g}^{t} \bar{P}_{g}, g \in G, t \in T$,

$$
f_{i, j}^{t}=B_{i, j}\left(\theta_{i}^{t}-\theta_{j}^{t}\right),(i, j) \in L, t \in T,
$$




$$
\begin{gathered}
-\bar{F}_{i, j} \leq f_{i, j}^{t} \leq \bar{F}_{i, j},(i, j) \in L, t \in T, \\
u_{g}^{t}-u_{g}^{t-1}=\nu_{g}^{t}-\mu_{g}^{t}, g \in G, t \in T, \quad \\
\sum_{i=t-T U_{g}+1}^{t} \nu_{g}^{i} \leq u_{g}^{t}, g \in G, t \in\left[T U_{g}, T\right] \\
\sum_{i=t-T D_{g}+1}^{t} \mu_{g}^{i} \leq 1-u_{g}^{t}, \\
\quad g \in G, t \in\left[T D_{g}, T\right], \quad \text { (12) } \\
-R D_{g} \leq p_{g}^{t}-p_{g}^{t-1} \leq R U_{g}, \\
\quad g \in G, t \in T, \\
\sum_{g \in G} r_{g}^{t} \geq \alpha^{t} \sum_{b \in B} L_{b}^{t}, t \in T, \\
u_{g}^{t}, \mu_{g}^{t}, \nu_{g}^{t} \in\{0,1\}, g \in G, t \in T,
\end{gathered}
$$

where inequalities (3)-(5) are linearizations of the total costs components; $C U_{g}, C P_{g}, C S_{g}, C D_{g}$, and $C R_{g}$, being the unit commitment cost, unit production cost, start-up cost, shut-down cost, and unit reserve cost of generator $g$, respectively; (6) is the joint load balance constraint; constraint (7) restricts the power generation and reserve contribution of generator $g$ to be within the minimum and maximum capacity in each period; (8) defines the line flow equations, and (9) bounds the power flowing through each transmission line; the logical constraint (10) links the UC status and start-up/shut-down decisions of the conventional generators. The minimum up/down time constraint (11)/(12) forces the generators to stay up/down for at least a specified number of time periods; the ramping up/down constraints are defined in (13); (14) guarantees the minimum reserves to be at least a fraction of the total net load in each time period; lastly, the commitment, start-up, and shut-down status of the generators are given by (15) for each time period.

A few remarks are in order here. First, the only source of uncertainty considered in this paper is wind resource, which does not affect the validity of our proposals. Indeed, the latter invokes no specific probability distribution assumptions on the net load process. Thus, other sources of uncertainty can be easily integrated in the model, without loss of generality. In addition, the modeling of the renewable energy or the net load processes is beyond the score of this work. Interested readers are referred to [30, 4, 31, 32] for further account.
On the other hand, we know that by Kirchhoff's first law, the supply and demand for energy must be balanced at all time, for the system to be in equilibrium. In (6), the load balance is instead formulated as an inequality, which is usually the case under a chance constraint framework, e.g., [29, 28, 13, 33, 34], as the probability of a continuous random variable taking on a single value is virtually zero. But, let us remind ourselves that the primary goal of the UC problem is to find the commitment status of the conventional generators for each time period. In real time, an economic dispatch problem is solved with the fixed commitment status of the generators to find the real time power outputs of the generators. Also, observe that technically speaking, since the production cost is minimized, no power will be produced more than needed. In optimal power flow analysis, other strategies exist. For instance, [16] uses an affine control to mitigate the fluctuation of power generation under wind energy integration by requiring each conventional generator production to be partially fixed and partially dependent on wind fluctuation.

\section{Approximation Approaches}

It is well known that chanced-constrained problems may be difficult to handle numerically, due to potential non-convexity of their feasible domains [35]. Except for special cases, e.g., under suitable distributional assumptions on the net load process [21, 22], it is no easy task deriving analytical expressions for the probabilistic load balance (6).

To circumvent this hurdle, two approximation schemes are presented in this work, namely (i) a robust relaxation of (6), discussed in Subsection 3.1; and (ii) a quantile approximation of (6), dealt with in Subsection 3.2. These two proposals will be benchmarked against the classical scenario-based approximation reviewed in Subsection 3.4 for the sake of completeness.

In a first step, instead of enforcing the load balance to jointly be met with probability no less than $\rho$ over the planning horizon and across the whole system, we shall require that in each period $t \in T$ the load balance be met with probability greater than $\rho$ at each bus $b \in B$, i.e.,

$$
\mathbb{P}\left(\psi_{b}^{t} \geq \hat{L}_{b}^{t}\right) \geq \rho, \quad b \in B, t \in T,
$$

where $\psi_{b}^{t}:=\sum_{g \in G_{b}}\left(p_{g}^{t}+r_{g}^{t}\right)+\sum_{l_{i, b} \in L} f_{i, b}^{t}-$ $\sum_{l_{b, j} \in L} f_{b, j}^{t}$, and $\hat{L}_{b}^{t}:=L_{b}^{t}-\sum_{i \in I_{b}} W_{i}^{t}$.

However, we recognize that the above implicit independence assumption of the nodal load balances may be deficient as (i) any two nodal equations containing the same network flow variable (e.g., for two neighboring nodes) may not be considered 
probabilistically independent; and (ii) loads at individual nodes may be driven by the same weather; similarly for wind generation. As a result, among other consequences, the relaxation of the joint load balance to individual counterparts no longer guarantees the reliability of the system as a whole. Thus, an iterative algorithm is proposed in Subsection 3.3 to adjust the individual confidence levels to the target joint reliability level, and by the same token restore the reliability of the whole network.

\subsection{Robust Approximation}

For each pair $b \in B, t \in T$, assume that the uncertain net load $\hat{L}_{b}^{t}$ depends upon a random variable $\xi_{b}^{t} \in \mathcal{U}_{b}^{t} \subset \mathbb{R}^{L}$ in an affine fashion; more specifically:

$$
\hat{L}_{b}^{t}\left(\xi_{b}^{t}\right)=\hat{L}_{b}^{0 t}+\sum_{l=1}^{L} \xi_{l, b}^{t} \hat{L}_{l, b}^{1 t}
$$

where $\hat{L}_{b}^{0 t}$ is a nominal net load, and the $\hat{L}_{l, b}^{1 t} \mathrm{~s}$ are known constant variations. We assume the scalar variates $\xi_{l, b}^{t} \mathrm{~s}$ to be zero-mean, independent (please, remember that the deficiency of this assumption will be addressed in Subsection 3.3), and with support $[-1,1]$. Under this setting, (16) is equivalent to:

$$
\mathbb{P}\left(\xi_{b}^{t^{\top}} \hat{L}_{b}^{1 t} \leq \psi_{b}^{t}-\hat{L}_{b}^{0 t}\right) \geq \rho, b \in B, t \in T
$$

On the other hand, suppose $\mathcal{U}_{b}^{t}$ to be the usual Euclidean ball, centered at zero, and with radius $\Omega_{b}^{t}$, i.e., $\mathcal{U}_{b}^{t}=\left\{\xi_{b}^{t}:\left\|\xi_{b}^{t}\right\|_{2} \leq \Omega_{b}^{t}\right\}$. It follows that (17) is the same as:

$$
\begin{array}{r}
\mathbb{P}\left(\xi_{b}^{t^{\top}} \hat{L}_{b}^{1 t} \leq \psi_{b}^{t}-\hat{L}_{b}^{0 t}:\left\|\xi_{b}^{t}\right\|_{2} \leq \Omega_{b}^{t}\right) \geq \rho, \\
b \in B, t \in T .
\end{array}
$$

Thus, for each period $t \in T$, and each bus $b \in B$, we want to find a robust feasible solution (to be guaranteed with probability no less than $\rho$ ), such that:

$$
\max _{\left\|\xi_{b}^{t}\right\|_{2} \leq \Omega_{b}^{t}} \xi_{b}^{t^{\top}} \hat{L}_{b}^{1 t} \leq \psi_{b}^{t}-\hat{L}_{b}^{0 t}
$$

Proposition 1. Constraint (20) is equivalent to:

$$
\psi_{b}^{t}-\hat{L}_{b}^{0 t} \geq \Omega_{b}^{t} \sqrt{\hat{L}_{b}^{1 t^{\top}} \hat{L}_{b}^{1 t}}
$$

For ease of notation, in the sequel, we drop all the indices.
Proof. Note that the left hand-side of (20) is the following quadratically constrained linear program:

$$
\begin{gathered}
\max _{\xi} \xi^{\top} \hat{L}^{1} \\
\text { subject to } \xi^{\top} \xi-\Omega^{2} \leq 0 .
\end{gathered}
$$

The Lagrangian function associated with Problem (22)-(23) is:

$$
L(\xi, \lambda):=-\xi^{\top} \hat{L}^{1}+\lambda\left(\xi^{\top} \xi-\Omega^{2}\right): \lambda \geq 0,
$$

and the corresponding dual function is:

$$
D(\lambda):=\max _{\xi}-\xi^{\top} \hat{L}^{1}+\lambda\left(\xi^{\top} \xi-\Omega^{2}\right): \lambda \geq 0 .
$$

In (25), taking the derivative w.r.t. $\xi$, and setting it to zero, we obtain:

$$
\xi=\frac{\hat{L}^{1}}{2 \lambda}, \forall \lambda \geq 0
$$

Now, since the objective function of (22)-(23) is linear, the maximum is attained at a boundary point, i.e., $\xi^{\top} \xi=$ $\Omega^{2}$. It is clear from (26) that $\lambda=\frac{\sqrt{\hat{L}_{b}^{1 t^{\top}} \hat{L}_{b}^{1 t}}}{2 \Omega}$, so that $\xi=\frac{\Omega \hat{L}^{1}}{\sqrt{\hat{L}_{b}^{1 t^{\top}} \hat{L}_{b}^{1 t}}}$. It is readily verified that the optimal value of (22)-(23), which is also the left hand-side of (20), is $\Omega \sqrt{\hat{L}_{b}^{1 t^{\top}} \hat{L}_{b}^{1 t}}$; and the proof is complete.

Lemma 1. [Azuma's inequality] Let $z \in \mathcal{R}^{L}$ be a deterministic vector, and $\xi_{1}, \ldots, \xi_{L}$ zero-mean random variables taking values in $[-1,1]$, then for any $\Omega>0$,

$$
\mathbb{P}\left(\xi^{\top} z>\Omega\|z\|_{2}\right) \leq \exp \left(-\Omega^{2} / 2\right) .
$$

Observe that (18) is the same as:

$$
\mathbb{P}\left(\xi_{b}^{t^{\top}} \hat{L}_{b}^{1 t}>\psi_{b}^{t}-\hat{L}_{b}^{0 t}\right) \leq 1-\rho, \quad b \in B, t \in T .
$$

It follows from the lemma that constraint (21) implies that:

$$
\begin{aligned}
\mathbb{P}\left(\xi_{b}^{t^{\top}} \hat{L}_{b}^{1 t}>\psi_{b}^{t}-\hat{L}_{b}^{0 t}\right) & \leq \mathbb{P}\left(\xi_{b}^{t^{\top}} \hat{L}_{b}^{1 t}>\Omega_{b}^{t} \sqrt{\hat{L}_{b}^{1 t^{\top}} \hat{L}_{b}^{1 t}}\right) \\
\leq & 1-\rho, \quad b \in B, t \in T
\end{aligned}
$$

provided that $1-\rho \geq \exp \left(-\Omega_{b}^{t^{2}} / 2\right)$ or $\Omega_{b}^{t} \geq$ $\sqrt{2 \ln \frac{1}{1-\rho}}$. As a result, the chance constraint (6) will be relaxed to the robust counterpart:

$$
\sum_{g \in G_{b}}\left(p_{g}^{t}+r_{g}^{t}\right)+\sum_{l_{i, b} \in L} f_{i, b}^{t}-\sum_{l_{b, j} \in L} f_{b, j}^{t} \geq \hat{L}_{b}^{0 t}+
$$




$$
\sqrt{2 \ln \frac{1}{1-\rho} \hat{L}_{b}^{1 t^{\top}} \hat{L}_{b}^{1 t}}, \quad b \in B, t \in T .
$$

In the numerical experiments, we will take the nominal net load, $\hat{L}_{b}^{0 t}$, as the sample mean, and the deviations, $\hat{L}_{b}^{1 t}$, as the sample standard deviation.

\subsection{A Quantile-based Approximation}

In contrast with the robust scheme, here, we invoke no assumption on the random net load $\hat{L}_{b}^{t}, b \in B, t \in$ $T$. We call $\hat{L}_{b, \rho}^{t}$ a $\rho$-percentile of the $(t, b)$-marginal distribution of the net load process, $t \in T, b \in B$, if $\mathbb{P}\left(V_{b}^{t} \leq V_{b, \rho}^{t}\right) \geq \rho$. As in [29], for each bus, in each time period, $\rho$-percentile estimates, $\tilde{\hat{L}}_{b, \rho}^{t}, t \in T, b \in B$, of $\hat{L}_{b, \rho}^{t}$, will be generated as follows.

Assume we have independent (potential consequences of this assumption are addressed in Subsection 3.3), identically distributed, and equally-likely observations (historical or generated), $\hat{L}_{b, 1}^{t}, \ldots, \hat{L}_{b, M}^{t}$, ordered as follows: $\hat{L}_{b,[1]}^{t} \leq \hat{L}_{b,[2]}^{t} \leq \ldots \leq \hat{L}_{b,[M]}^{t}$. Using $\hat{L}_{b,[\rho M\rceil}^{t}$, $\lceil\cdot\rceil$ being the ceiling function, as a point estimate of $\tilde{\hat{L}}_{b, \rho}^{t}, t \in T, b \in B$, we have the following quantile relaxation of (6):

$$
\begin{gathered}
\sum_{g \in G_{b}}\left(p_{g}^{t}+r_{g}^{t}\right)+\sum_{l_{i, b} \in L} f_{i, b}^{t}-\sum_{l_{b, j} \in L} f_{b, j}^{t} \geq \\
\hat{L}_{b,\lceil\rho M\rceil}^{t}, \quad b \in B, t \in T .
\end{gathered}
$$

\subsection{Iterative Adjustment of the Individual Confidence Levels}

As will be illustrated in the numerical experiments, enforcing (30) and (31) with high probability does not guarantee that the joint chance constraint (6) will be met with the same probability. This is well known in the literature of chance-constrained programming. As a result, the robust and quantile approximations may yield unreliable solutions for the operation of the power system as a whole.

To mitigate this issue, we will use the following iterative procedure, inspired by [13], but without any distributional assumption on the net load, to find sample risk values $\phi$ of each individual probabilistic constraint (at each time period and for each bus) until the joint load balance (over the planning horizon and across the power system) is met above the prescribed reliability level $\rho$.
In (16), denote the event $\psi_{b}^{t} \geq \hat{L}_{b}^{t}, b \in B, t \in T$, by $E_{b}^{t}$, and its complement $\psi_{b}^{t}<\hat{L}_{b}^{t}, b \in B, t \in T$, by $E_{b}^{t^{\prime}}$. From the finite subadditivity of $\mathbb{P}$, also called Boole's inequality, it is well known that:

$$
\mathbb{P}\left(\bigcup_{b \in B, t \in T} E_{b}^{t^{\prime}}\right) \leq \sum_{b \in B, t \in T} \mathbb{P}\left(E_{b}^{t^{\prime}}\right)
$$

For each pair $b \in B, t \in T$, if we take $\mathbb{P}\left(E_{b}^{t^{\prime}}\right) \leq$ $(1-\rho) /(|B| \times|T|)$, it follows immediately from (32) that:

$$
\begin{aligned}
\mathbb{P}\left(\bigcap_{b \in B, t \in T} E_{b}^{t^{\prime}}\right) & =1-\mathbb{P}\left(\bigcup_{b \in B, t \in T} E_{b}^{t^{\prime}}\right) \\
& \geq 1-\sum_{b \in B, t \in T} \mathbb{P}\left(E_{b}^{t^{\prime}}\right) \\
& \geq 1-(1-\rho) /(|B| \times|T|) .
\end{aligned}
$$

As a result, we choose our initial confidence level as $\phi_{0}=1-(1-\rho) /(|B| \times|T|)$.

The iterative scheme proceeds as follows. First, we choose a lower bound, $\underline{\phi}$, of $\phi$, e.g., $\rho$, as well as an upper bound, $\bar{\phi}$, (e.g., a very high probability). Under the robust approximation, we solve the UC problems by substituting $\rho$ with $\phi_{0}, \underline{\phi}$, and $\bar{\phi}$, respectively in (30). In the quantile approximation, UC problems are solved with the estimated net load quantiles $\hat{L}_{b,\left\lceil\phi_{0} M\right\rceil}^{t}, \hat{L}_{b,\lceil\underline{\phi} M\rceil}^{t}$, and $\hat{L}_{b,\lceil\bar{\phi} M\rceil}^{t}$, respectively (constraint (31)).

Next, the load balances - for all time periods and all the buses - are checked to find the empirical joint probabilities, $\rho_{k}, \underline{\rho}$, and $\bar{\rho}$, respectively. If $\rho_{k}$ is higher than $\rho$, and lower than $\rho$, we increase the lower bound, $\phi$, to the current value of $\phi, \phi_{k}$; otherwise, if $\rho_{k}$ is higher than $\rho$, and lower than $\bar{\rho}$, we decrease the upper bound, $\bar{\phi}$, to $\phi_{k}$. Lastly, we interpolate the individual confidence levels $\phi$ as follows:

$$
\phi_{k}=\underline{\phi}+\left(\frac{\rho-\underline{\rho}}{\bar{\rho}-\underline{\rho}}\right)(\bar{\phi}-\underline{\phi}) .
$$

This iterative scheme is repeated until the difference between the empirical joint probability $\rho_{k}$ and the desired value $\rho$ is less than a fixed tolerance. 


\subsection{Scenario-based Approximation}

Here, assume the multivariate stochastic net load process to be discrete and finite, and the realizations to be indexed in the set $S$. Without loss of generality, the probability of each scenario $s \in S$ is taken as $p^{s}=1 /|S|$. In period $t$, the net load at bus $b$ under scenario $s$ is denoted $\hat{L}_{b}^{t, s}$. For more account of wind speed/power generation, refer to [36, 31, 37, 38].

We want to enforce the load balance constraint (6) for a subset of the scenarios indexed in $S_{\rho} \subseteq S$ such that $\sum_{s \in S_{\rho}} p^{s} \geq \rho$. This will be achieved through the following additional binary variables:

$\delta^{s}= \begin{cases}1 & \text { if all constraints in scenario } s \text { are satisfied } \\ 0 & \text { otherwise. }\end{cases}$

The probabilistic load balance (6) is relaxed as follows:

$$
\begin{aligned}
& \sum_{g \in G_{b}}\left(p_{g}^{t}+r_{g}^{t}\right)+\sum_{l_{i, b} \in L} f_{i, b}^{t}-\sum_{l_{b, j} \in L} f_{b, j}^{t} \geq \\
& \quad \max \left(0, \hat{L}_{b}^{t, s}\right) \delta^{s}, b \in B, t \in T, s \in S, \\
& \sum_{s \in S} p^{s} \delta^{s} \geq \rho, \\
& \delta^{s} \in\{0,1\}, s \in S .
\end{aligned}
$$

The resulting approximate scenario UC problem may be a large-scale MIP due the size of the set $S$, and as a result may be intractable for even modest sample sizes due to the knapsack constraint (35). To alleviate the computational burden of the resulting MIP, a decomposition approach is proposed. A lower bound, called quantile bound is obtained by solving the MIP for each scenario $s \in S$, with $\delta^{s}=1$, and relaxing the knapsack constraint (35).

Let $f_{s}, s \in S$, be the optimal value of the UC problem under scenario $s$. Then, sort the $|S|$ optimal values to obtain a permutation $\eta$ of $S$ such that $f_{\eta_{1}} \leq$ $f_{\eta_{2}} \cdots \leq f_{\eta_{S}}$. The quantile bound is then taken as $V^{Q}:=f_{\eta_{q}}$, where $q:=\min \left\{k \in S: \sum_{i=1}^{k} p^{\eta_{i}} \geq \rho\right\}$. This clearly provides a lower bound for the approximate scenario UC problem, as at least one scenario of the permutation $\eta:=\left\{\eta_{1}, \cdots, \eta_{q}\right\}$ is satisfied in a feasible solution [39].

Observe that the resulting UC solutions to the scenario sub-problems are scenario-dependent, and as a result are no longer unique or "non-anticipative". However, the lower bound, $V^{Q}$, will still be useful to benchmark the optimal values of our approximate problems. This limitation could be tackled through the popular progressive hedging algorithm of [40], as applied in [41], but at the expense of more demanding computations than the simple quantile bound approximation. For other decomposition strategies, please see [42, 39].

\section{Numerical Experiments}

The test problems are briefly described in Subsection 4.1, and their results are reported in Subsection 4.2.

\subsection{Experimental Framework}

Our test cases comprise the 5-bus system of the AMES wholesale power market test bed, the IEEE 30and 118-bus systems. The AMES 5-bus system has 5 buses, 5 generators, 6 transmission lines, and a wind farm hosted at bus 2. A scheduling horizon of 24 hours, divided in hourly time steps, is considered. The load profile is displayed in Figure 1a and an example of 10 wind scenarios plotted in Figure $1 \mathrm{~b}$. The IEEE 30-bus system consists of 30 buses, 6 generators, 41 transmission lines with a wind farm hosted at bus 2 . The IEEE 118-bus system consists of 118 buses, 54 generators, 186 transmission lines, and a wind farm located at bus 6 .

The solution algorithms were implemented in Python 3.6 and the optimization programs modeled in Pyomo, an open-source software package for modeling and solving optimization programs. ILOG CPLEX 12.8 was employed to solve the resulting mixed-integer linear optimization programs. All the experiments were conducted on a computer featuring a $2.9 \mathrm{GHz}$ Intel Core and 8 GB memory.

\subsection{Results}

The following lines of inquiry are pursued. In Subsection 4.2.1, we analyze the operating costs across the approximation methods when individual confidence levels are set on the load balance; in Subsection 4.2.2, we present the operating costs when a joint confidence level is sought for the whole system over the planning horizon, under the robust and the quantile schemes; lastly, the three approximation methods are compared based on their associated computation time in Subsection 4.2.3.

\subsubsection{Operating Costs: Individual Reliability} Levels We are interested in the fraction of the total load that is satisfied for different confidence levels $\rho$, and the variation of the resulting UC costs across the approximation methods. Under the scenario-based approximation, the value of the reliability level $\rho$ 


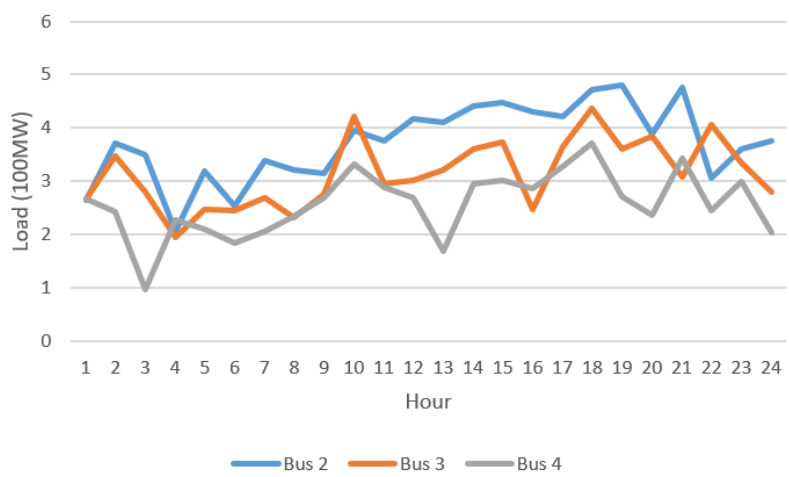

(a) Load Profile of 5-Bus Instance

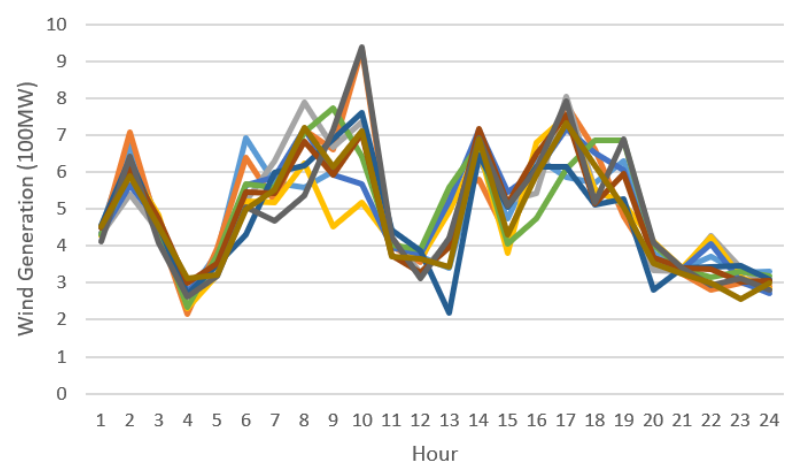

(b) An Example of 10 Wind Scenarios

Figure 1: Load and Wind Profile of the AMES 5-Bus System

represents the fraction of the scenarios in which the load balance constraints are satisfied for all the time periods and all buses, as in (35); while under both the robust and the quantile-based approximation methods, the value $\rho$ corresponds to the confidence level of the load constraint for each bus and each period, as depicted in constraints (30), and (31), respectively. The resulting approximate UC costs are displayed in Figure 2. In addition, 100 wind scenarios are randomly generated from a normal distribution to evaluate the percentage of scenarios in which electricity loads are satisfied at all the buses over the entire planning horizon, which is also shown in Figure 2.

As illustrated in Figure 2, the robust scheme clearly provides a better approximation of the scenario-based scheme while the quantile-based approximation consistently yields a lower bound. This can be explained by the resulting percentage of scenarios for which the load balance constraints are jointly satisfied. As the confidence level, $\rho$, decreases from 0.99 to 0.9 , the percentage of satisfied scenarios resolved from the quantile-based approximation decreases dramatically to

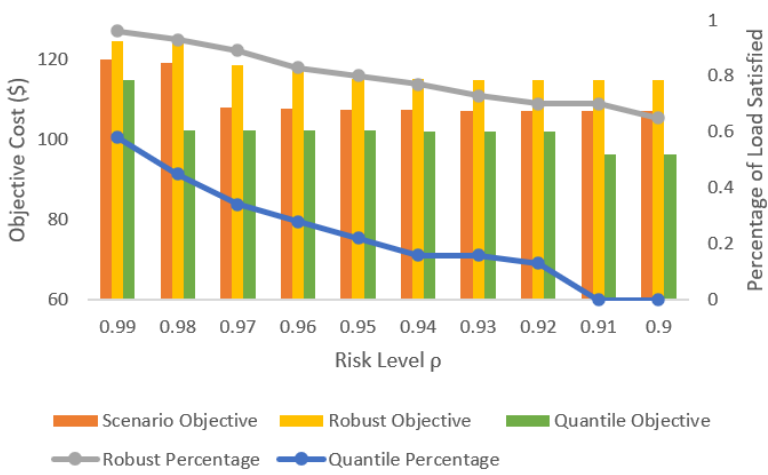

Figure 2: UC Operating Costs (\$) and Fraction of the Satisfied Load with Different Values of $\rho$

0 , resulting in a much lower objective costs compared to the scenario-based counterpart. This suggests that the individual risk levels need to be uplifted significantly in order to reach the target joint confidence level. We analyze the effect of the iterative adjustment heuristic discussed in Subsection 3.3 next.

\subsubsection{Operating Costs: Joint Reliability Level} In this section, we compare the three approximation schemes with regard to their UC operating costs given varying target risk levels for the joint load balance constraint. Under the scenario-based model, we simply assign the target value $\rho$ to constraint (35). Under both the robust- and the quantile-based approximation, we use the iterative algorithm to update the individual confidence level for each bus and each period until the percentage of satisfied joint load constraints is close enough to the target $\rho$. The optimal costs yielded by the different approximation schemes are depicted in Figure 3, which also illustrates the individual confidence levels that lead to the target confidence level. Here, the same set of 500 wind scenarios are used under the three approximation methods.

As can be seen from Figure 3a, both the robustand the quantile-based approximation schemes yield higher operating costs compared to the scenario-based approximation counterpart, as a result of shrunk feasible regions from the additional requirements that all the individual load balance constraints share the same confidence level. Compared to the quantile-based scheme, as the individual confidence levels decrease, the robust model leads to a better approximation of the scenario-problem, which is also suggested by the individual reliability levels displayed in Figure 3. While in the robust models, the individual confidence levels decrease significantly as 

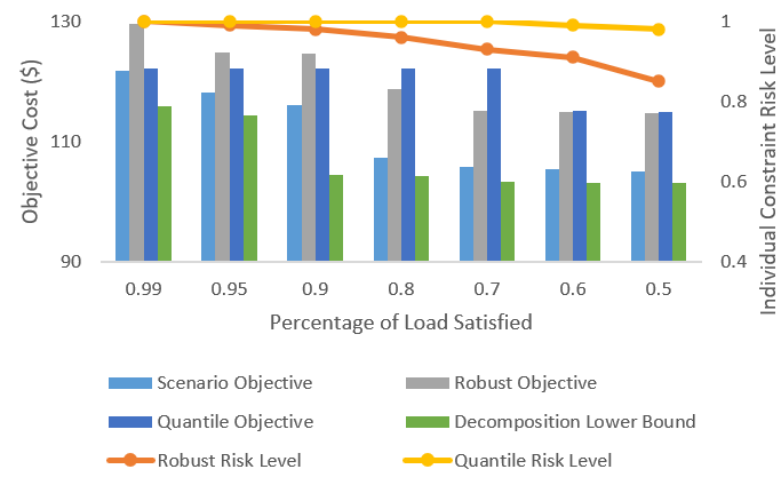

(a) AMES 5-Bus System

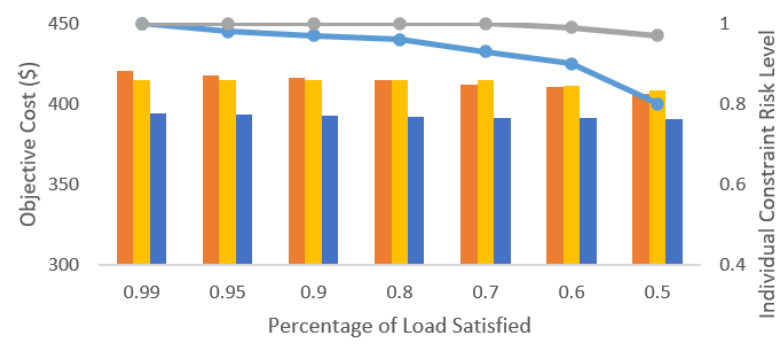

Robust Objective Quantile Objective

Decomposition Lower Bound - Robust Risk Level

-Quantile Risk Level

(b) IEEE 30-Bus System

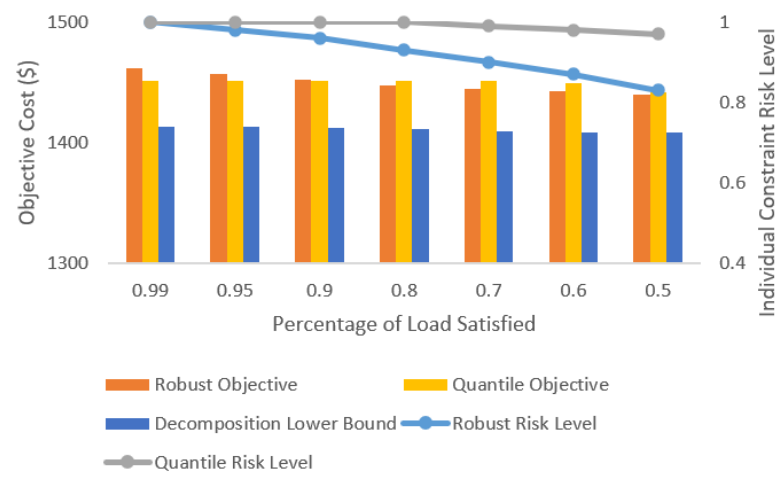

(c) IEEE 118-Bus System

Figure 3: UC Costs (\$) and Individual Constraint Confidence Levels of $\rho$ with Different Target Percentage of Load Satisfied

the fraction of satisfied load constraints does, under the quantile-based approximation, the individual risk levels barely change over the percentage of satisfied load constraints and stay close to $100 \%$ in all three test cases.

Due to the large number of binary variables, the operating costs of the scenario-based approximation are not available for larger scales of UC problems, e.g., IEEE 30- and IEEE 118-bus systems as in Figures 3b and $3 \mathrm{c}$. Thus, the decomposition strategy is adopted to provide a lower bound of the scenario-based model to benchmark the robust and quantile-based schemes. This lower bound further demonstrates the tightness of the upper bounds obtained from the robust- and quantile-based models.

4.2.3. Runtime Furthermore, the runtimes of the robust and the quantile approximation approaches were benchmarked against the classical scenario approximation and its decomposition strategy on all three test systems with varying number of wind scenarios and a risk-level of $\rho=0.9$, as displayed in Table 1. The computation was terminated if the test instance was not solved within an hour; such cases are labeled as N/A in Table 1.

As shown in Table 1, the runtime of the scenario-based method increased dramatically with the sample size, especially in large scale test cases; the scheme failed to solve the 5-bus instance with more than 1,000 scenarios and the IEEE 118-bus test case with more than 100 scenarios within an hour. This computational burden is the result of duplicating the scenario-wise variables and constraints for each scenario. When the sample size is large, the size of the problem significantly increases. The runtimes from the decomposition strategy, as shown in different test scales, are consistently proportional to the number of scenarios. That is because this strategy decomposes the scenario-based model into scenario sub-problems and then solves a deterministic UC problem for each scenario sequentially.

On the contrary, the runtime of the robust and the quantile-based approximations vary very little across different sample sizes for all test systems. This is because both the robust and the quantile-based approximation solve a set of deterministic optimization problems with the same number of variables and constraints regardless of the sample size. As the number of scenarios increases, the increment in the computational burden only amounts to checking the load balance constraints for each scenario, which is negligible compared to the computational burden of solving the optimization programs. The results illustrate the computational efficiency of the robustand the quantile-based approximations over the classical scenario-based counterpart, especially for large-scale systems.

\section{Conclusions}

This paper analyzed a multi-period unit commitment problem under the integration of wind power. Due 


\begin{tabular}{|l|l|l|l|l|}
\hline $\begin{array}{l}\text { Number of } \\
\text { Scenarios }\end{array}$ & Scenario & Robust & Quantile & Decomposition \\
\hline 10 & 7 & 13 & 12 & 23 \\
\hline 100 & 65 & 14 & 14 & 178 \\
\hline 200 & 417 & 15 & 14 & 344 \\
\hline 500 & 2,837 & 17 & 18 & 793 \\
\hline 1,000 & N/A & 16 & 17 & 1482 \\
\hline
\end{tabular}

(a) AMES 5-Bus System

\begin{tabular}{|l|l|l|l|l|}
\hline $\begin{array}{l}\text { Number of } \\
\text { Scenarios }\end{array}$ & Scenario & Robust & Quantile & Decomposition \\
\hline 10 & 7 & 18 & 22 & 16 \\
\hline 30 & 46 & 18 & 23 & 44 \\
\hline 50 & 358 & 18 & 25 & 82 \\
\hline 100 & 1,770 & 17 & 22 & 238 \\
\hline 200 & N/A & 25 & 24 & 477 \\
\hline
\end{tabular}

(b) IEEE 30-Bus System

\begin{tabular}{|l|l|l|l|l|}
\hline $\begin{array}{l}\text { Number of } \\
\text { Scenarios }\end{array}$ & Scenario & Robust & Quantile & Decomposition \\
\hline 10 & 26 & 50 & 40 & 49 \\
\hline 20 & 208 & 53 & 85 & 90 \\
\hline 30 & 279 & 78 & 43 & 131 \\
\hline 50 & 3,509 & 70 & 56 & 222 \\
\hline 100 & N/A & 73 & 55 & 486 \\
\hline
\end{tabular}

(c) IEEE 118-Bus System

Table 1: Run-time (seconds) Comparison

to the high volatility of the latter, the load balance was enforced with a desired confidence level over the planning horizon and across the power network. As, in general, chance-constraints are recognized to be difficult to handle numerically, this work presented two approximation schemes to relax the joint probabilistic load balance. To alleviate the potential operational consequences of the relaxations, an iterative scheme aiming at adjusting the individual confidence levels to the desired joint confidence level was also delineated.

Both the proposed robust- and quantile-based approximations provide an upper bound of the scenario-based model within a significantly shorter time frame. In addition, both approximation approaches are distribution free. In terms of solution quality, both schemes provide good approximations of the problem as benchmarked against a lower bound of the scenario-based model. From the perspective of computational efficiency, both schemes display remarkable advantages over the classical scenario-based model, especially on large-scale systems as the associated runtime varies little with increasing sample sizes. Lastly, overall, the quantile scheme yields reliability levels closer to the predefined confidence levels of the load balance constraints, which might provide an edge against the robust-based approximation.

\section{References}

[1] Z. Wang and C. L. Anderson, "A progressive period optimal power flow for systems with high penetration of variable renewable energy sources," Energies, vol. 14, no. 10, p. 2815, 2021.

[2] Z. Wang and Z. Guo, "On critical timescale of real-time power balancing in power systems with intermittent power sources," Electric Power Systems Research, vol. 155, pp. 246-253, 2018.

[3] Z. Wang, G. Guo, and C. L. Anderson, "Simulation case studies on period optimal power flow," in 2019 Winter Simulation Conference (WSC), pp. 3669-3680, IEEE, 2019.

[4] A. Papavasiliou, S. S. Oren, and R. P. O'Neill, "Reserve requirements for wind power integration: A scenario-based stochastic programming framework," IEEE Transactions on Power Systems, vol. 26, no. 4, pp. 2197-2206, 2011.

[5] G. Guo, L. Zephyr, J. Morillo, Z. Wang, and C. L. Anderson, "Chance constrained unit commitment approximation under stochastic wind energy," Computers \& Operations Research, p. 105398, 2021.

[6] L. Zéphyr and C. L. Anderson, "Stochastic dynamic programming approach to managing power system uncertainty with distributed storage," Computational Management Science, vol. 15, no. 1, pp. 87-110, 2018.

[7] J. Cardell and C. Anderson, "Targeting existing power plants: Epa emission reduction with wind and demand response," Energy Policy, vol. 80, pp. 11-23, 2015.

[8] R. Jiang, J. Wang, and Y. Guan, "Robust unit commitment with wind power and pumped storage hydro," IEEE Transactions on Power Systems, vol. 27, no. 2, pp. 800-810, 2012.

[9] D. Bertsimas, E. Litvinov, X. A. Sun, J. Zhao, and T. Zheng, "Adaptive robust optimization for the security constrained unit commitment problem," IEEE transactions on power systems, vol. 28 , no. 1, pp. 52-63, 2012.

[10] C. Zhao, J. Wang, J.-P. Watson, and Y. Guan, "Multi-stage robust unit commitment considering wind and demand response uncertainties," IEEE Transactions on Power Systems, vol. 28, no. 3, pp. 2708-2717, 2013.

[11] C. Zhao and Y. Guan, "Unified stochastic and robust unit commitment," IEEE Transactions on Power Systems, vol. 28, no. 3, pp. 3353-3361, 2013.

[12] Q. Wang, Y. Guan, and J. Wang, "A chance-constrained two-stage stochastic program for unit commitment with uncertain wind power output," IEEE Transactions on Power Systems, vol. 27, no. 1, pp. 206-215, 2012.

[13] U. A. Ozturk, M. Mazumdar, and B. A. Norman, "A solution to the stochastic unit commitment problem using chance constrained programming," IEEE Transactions on Power Systems, vol. 19, no. 3, pp. 1589-1598, 2004.

[14] C. Zhao, Q. Wang, J. Wang, and Y. Guan, "Expected value and chance constrained stochastic unit commitment ensuring wind power utilization," IEEE Transactions on Power Systems, vol. 29, no. 6, pp. 2696-2705, 2014.

[15] Z. Wu, P. Zeng, X.-P. Zhang, and Q. Zhou, "A solution to the chance-constrained two-stage stochastic program for unit commitment with wind energy integration," IEEE Transactions on Power Systems, vol. 31, no. 6, pp. 4185-4196, 2016.

[16] D. Bienstock, M. Chertkov, and S. Harnett, "Chance-constrained optimal power flow: Risk-aware network control under uncertainty," Siam Review, vol. 56, no. 3, pp. 461-495, 2014. 
[17] L. Roald, S. Misra, M. Chertkov, and G. Andersson, "Optimal power flow with weighted chance constraints and general policies for generation control," in 2015 54th IEEE conference on decision and control (CDC), pp. 6927-6933, IEEE, 2015.

[18] D. Pozo and J. Contreras, "A chance-constrained unit commitment with an $n k$ security criterion and significant wind generation," IEEE Transactions on Power systems, vol. 28, no. 3, pp. 2842-2851, 2013.

[19] P. Xiong and P. Jirutitijaroen, "A stochastic optimization formulation of unit commitment with reliability constraints," IEEE Transactions on Smart Grid, vol. 4, no. 4, pp. 2200-2208, 2013.

[20] Y. Zhang, J. Wang, B. Zeng, and Z. Hu, "Chance-constrained two-stage unit commitment under uncertain load and wind power output using bilinear benders decomposition," IEEE Transactions on Power Systems, vol. 32, no. 5, pp. 3637-3647, 2017.

[21] J. L. Morillo, J. F. Pérez, L. Zéphyr, C. L. Anderson, and A. Cadena, "Assessing the impact of wind variability on the long-term operation of a hydro-dominated system," in 2017 IEEE PES Innovative Smart Grid Technologies Conference Europe (ISGT-Europe), pp. 1-6, IEEE, 2017.

[22] J. L. Morillo, L. Zéphyr, J. F. Perez, C. L. Anderson, and Á. Cadena, "Risk-averse stochastic dual dynamic programming approach for the operation of a hydro-dominated power system in the presence of wind uncertainty," International Journal of Electrical Power \& Energy Systems, vol. 115, p. 105469, 2020.

[23] L. Hongling, J. Chuanwen, and Z. Yan, "A review on risk-constrained hydropower scheduling in deregulated power market," Renewable and Sustainable Energy Reviews, vol. 12, no. 5, pp. 1465-1475, 2008.

[24] R. Jiang and Y. Guan, "Data-driven chance constrained stochastic program," Mathematical Programming, vol. 158 , no. $1-2$, pp. 291-327, 2016.

[25] B. A. Calfa, I. E. Grossmann, A. Agarwal, S. J. Bury, and J. M. Wassick, "Data-driven individual and joint chance-constrained optimization via kernel smoothing," Computers \& Chemical Engineering, vol. 78, pp. 51-69, 2015.

[26] H. Zhang, Z. Hu, E. Munsing, S. J. Moura, and Y. Song, "Data-driven chance-constrained regulation capacity offering for distributed energy resources," IEEE Transactions on Smart Grid, 2018.

[27] X. Ding, W.-J. Lee, W. Jianxue, and L. Liu, "Studies on stochastic unit commitment formulation with flexible generating units," Electric power systems research, vol. 80 , no. 1 , pp. $130-141,2010$.

[28] G. Martínez and L. Anderson, "A risk-averse optimization model for unit commitment problems," in 2015 48th Hawaii International Conference on System Sciences, pp. 2577-2585, IEEE, 2015.

[29] G. Martínez and L. Anderson, "Toward a scalable chance-constrained formulation for unit commitment to manage high penetration of variable generation," in 2014 52nd Annual Allerton Conference on Communication, Control, and Computing (Allerton), pp. 723-730, IEEE, 2014.

[30] L. Fortuna, G. Nunnari, and S. Nunnari, Nonlinear modeling of solar radiation and wind speed time series. Springer, 2016.
[31] K. C. Sharma, P. Jain, and R. Bhakar, "Wind power scenario generation and reduction in stochastic programming framework," Electric Power Components and Systems, vol. 41, no. 3, pp. 271-285, 2013.

[32] M. Alipour, J. Aghaei, M. Norouzi, T. Niknam, S. Hashemi, and M. Lehtonen, "A novel electrical net-load forecasting model based on deep neural networks and wavelet transform integration," Energy, vol. 205, p. 118106, 2020.

[33] X. Geng and L. Xie, "Chance-constrained unit commitment via the scenario approach," in 2019 North American Power Symposium (NAPS), pp. 1-6, IEEE, 2019.

[34] Q. Wang, Y. Guan, and J. Wang, "A chance-constrained two-stage stochastic program for unit commitment with uncertain wind power output," IEEE Transactions on Power Systems, vol. 27, no. 1, pp. 206-215, 2011.

[35] B. Liu, "Theory and practice of uncertain programming. wurzburg, berlin: Physica," 2002.

[36] X.-Y. Ma, Y.-Z. Sun, and H.-L. Fang, "Scenario generation of wind power based on statistical uncertainty and variability," IEEE Transactions on Sustainable Energy, vol. 4, no. 4, pp. 894-904, 2013.

[37] J. Sumaili, H. Keko, V. Miranda, Z. Zhou, A. Botterud, and J. Wang, "Finding representative wind power scenarios and their probabilities for stochastic models," in 2011 16th International Conference on Intelligent System Applications to Power Systems, pp. 1-6, IEEE, 2011.

[38] J. M. Morales, R. Minguez, and A. J. Conejo, "A methodology to generate statistically dependent wind speed scenarios," Applied Energy, vol. 87, no. 3, pp. 843-855, 2010.

[39] S. Ahmed, J. Luedtke, Y. Song, and W. Xie, "Nonanticipative duality, relaxations, and formulations for chance-constrained stochastic programs," Mathematical Programming, vol. 162, no. 1-2, pp. 51-81, 2017.

[40] R. T. Rockafellar and R. J.-B. Wets, "Scenarios and policy aggregation in optimization under uncertainty," Mathematics of operations research, vol. 16, no. 1, pp. 119-147, 1991.

[41] J.-P. Watson, R. J. Wets, and D. L. Woodruff, "Scalable heuristics for a class of chance-constrained stochastic programs," INFORMS Journal on Computing, vol. 22, no. 4, pp. 543-554, 2010.

[42] S. Ahmed and W. Xie, "Relaxations and approximations of chance constraints under finite distributions," Mathematical Programming, vol. 170, no. 1, pp. 43-65, 2018 . 